Mathematical Modelling AND ANALysis

Volume 18 Number 4, September 2013, 480-488 http://dx.doi.org/10.3846/13926292.2013.841298

(c) Vilnius Gediminas Technical University, 2013
Publisher: Taylor\&Francis and VGTU

http://www.tandfonline.com/TMMA

Print ISSN: 1392-6292

Online ISSN: 1648-3510

\title{
Properties of Zeros of Solutions to Third Order Nonlinear Differential Equations
}

\section{Sergey Smirnov}

\author{
Institute of Mathematics and Computer Science, University of Latvia \\ Raina bulvaris 29, LV-1459 Riga, Latvia \\ E-mail: srgsm@inbox.lv
}

Received September 14, 2012; revised July 26, 2013; published online September 1, 2013

\begin{abstract}
We investigate the behavior of zeros of solutions to the certain type of third order nonlinear differential equations. We show that the behavior of zeros may be rather different and depend on the nature of nonlinearity in the equation. Main results in the paper are illustrated with a number of examples.
\end{abstract}

Keywords: third order nonlinear differential equations, zeros of solutions, dependence of zeros on initial data, super-linearity, sub-linearity.

AMS Subject Classification: $34 \mathrm{C} 10$.

\section{Introduction}

Consider a third order nonlinear differential equation of the form

$$
x^{\prime \prime \prime}=-\lambda p(t) f(x)
$$

where $\lambda$ is a positive parameter, functions $f$ and $p$ are continuous, $p(t) \geq 0$, $p(t) \not \equiv 0$. We are interested in solutions of equation (1.1) which satisfy the initial conditions

$$
x(0)=x^{\prime}(0)=0, \quad x^{\prime \prime}(0)=\beta,
$$

where $\beta$ is also a parameter. It is our goal to study zeros of solutions as functions of parameters $\lambda$ and $\beta$.

We will use also the following assumptions:

(H1) $\quad x f(x)>0$ for $x \neq 0$;

(H2) $\quad p(B t)=B^{k} p(t)$ for some $k \geq 0$ and all $B>0$;

(H3) $f(B x)=B^{q} f(x)$ for some $q>0, q \neq 1$ and all $B>0$.

The existence, location and properties of the zeros of the solutions of ordinary differential equations are of principal importance in the theory of boundary value problems $[5,6,7]$, and correspondingly a vast literature on this subject has 
arisen. Among numerous papers dealing with this subject we refer the reader in particular to $[2,3]$ for linear equations, and $[1,4,8]$ for nonlinear equations.

The paper is organized as follows. Section 2 contains some auxiliary results. Section 3 is devoted to the oscillatory properties of solutions of equation (1.1). In Section 4 we consider dependence of zeros of solutions on parameters. Also examples are given to illustrate the results.

\section{Preliminary Results}

Proposition 1. Suppose $x(t) \in C^{3}(I)$. If $x(a) \geq 0, x^{\prime}(a) \leq 0, x^{\prime \prime}(a) \geq 0$ (but not all zero) and $x^{\prime \prime \prime}(t) x(t)<0$ when $x(t) \neq 0$, then $x(t)>0, x^{\prime}(t)<0$, $x^{\prime \prime}(t)>0$ for $t<a$.

Proof. Let $x(a) \geq 0, x^{\prime}(a) \leq 0, x^{\prime \prime}(a) \geq 0$ and $(x(a))^{2}+\left(x^{\prime}(a)\right)^{2}+\left(x^{\prime \prime}(a)\right)^{2}>0$. In all cases $x(t)$ will be positive in some open interval with the right boundary point $t=a$. Suppose that there exists a point $t=t_{0}$ such that $x\left(t_{0}\right)=0$ and $x(t)>0$ for $t_{0}<t<a$. Since $x\left(t_{0}\right)=0$, there will exist a point $t=t_{1}$, $t_{0} \leq t_{1}<a$ such that $x^{\prime}\left(t_{1}\right)=0$ and there will exist a point $t=t_{2}, t_{0} \leq t_{2}<a$ such that $x^{\prime \prime}\left(t_{2}\right)=0$. Since $x^{\prime \prime \prime}(t) x(t) \leq 0$, it follows that $x^{\prime \prime \prime}(t)<0$ for $t_{0}<t<a$. Let us consider

$$
x^{\prime \prime}(t)=x^{\prime \prime}(a)-\int_{t}^{a} x^{\prime \prime \prime}(s) d s, \quad t_{0} \leq t<a .
$$

The right-hand side is positive, and increases as $t \rightarrow-\infty$, as long as $x^{\prime \prime \prime}(t)$ remains negative. We thus conclude that $x^{\prime \prime}(t)$ is positive for $t_{0} \leq t<a$.

Let us consider

$$
x^{\prime}(t)=x^{\prime}(a)-\int_{t}^{a} x^{\prime \prime}(s) d s, \quad t_{0} \leq t<a .
$$

The right-hand side is negative, and decreases as $t \rightarrow-\infty$, as long as $x^{\prime \prime}(t)$ remains positive. We thus conclude that $x^{\prime}(t)$ is negative for $t_{0} \leq t<a$.

Let us consider

$$
x(t)=x(a)-\int_{t}^{a} x^{\prime}(s) d s, \quad t_{0} \leq t<a .
$$

The right-hand side is positive, and increases as $t \rightarrow-\infty$, as long as $x^{\prime}(t)$ remains negative. We thus conclude that $x(t)$ is positive for $t_{0} \leq t<a$. These contradictions prove the proposition.

Corollary 1. Suppose $x(t) \in C^{3}(I)$. If $x(a) \leq 0, x^{\prime}(a) \geq 0, x^{\prime \prime}(a) \leq 0$ (but not all zero) and $x^{\prime \prime \prime}(t) x(t)<0$ when $x(t) \neq 0$, then $x(t)<0, x^{\prime}(t)>0, x^{\prime \prime}(t)<0$ for $t<a$.

Proof. The proof follows from Proposition 1 considering $y(t)=-x(t)$.

Remark 1. The function $x(t)$ from Proposition 1 and Corollary 1 may be assumed to be a solution of differential equation (1.1). 
Proposition 2. If a function $f(x)$ satisfies assumptions (H1) and (H3), then $f(x)$ is strictly increasing.

Proof. Let us choose $0<x_{1}<x_{2}$. Obviously, there exists a $B>1$ such that $x_{2}=B x_{1}$. Now consider $f\left(x_{2}\right)=f\left(B x_{1}\right)=B^{q} f\left(x_{1}\right)>f\left(x_{1}\right)$ (we use the assumption $x f(x)>0$ to obtain the last inequality). Thus, $f(x)$ is strictly increasing function for $x>0$. Analogously we can show that $f(x)$ is strictly increasing for $x<0$. Let $x_{2}<x_{1}<0$. Then there exists a $B>1$ such, that $x_{2}=B x_{1}$. Now consider $f\left(x_{2}\right)=f\left(B x_{1}\right)=B^{q} f\left(x_{1}\right)<f\left(x_{1}\right)$. Thus $f(x)$ is strictly increasing function for $x<0$.

Proposition 3. Suppose, that a function $p(t)$ satisfies assumption $(\mathrm{H} 2)$. If $k>0$, then $p(t)$ is increasing for $t>0$ and decreasing for $t<0$. If $k=0$, then $p(t)=$ Const $>0$.

Proof. Assume $k>0$. First, let us prove that the function $p(t)$ is increasing for $t>0$. Let us choose $0<t_{1}<t_{2}$. Obviously, there exists a $B>1$ such that $t_{2}=B t_{1}$. Now consider $p\left(t_{2}\right)=p\left(B t_{1}\right)=B^{k} p\left(t_{1}\right) \geq p\left(t_{1}\right)$ (we use the assumption $p(t) \geq 0$ to obtain the last inequality). Thus, $p(t)$ is an increasing function for $t>0$. Next, let us prove that the function $p(t)$ is decreasing for $t<0$. Let us choose $t_{2}<t_{1}<0$. Obviously, there exists a $B>1$ such that $t_{2}=B t_{1}$. Now consider $p\left(t_{2}\right)=p\left(B t_{1}\right)=B^{k} p\left(t_{1}\right) \geq p\left(t_{1}\right)$. Thus $p(t)$ is decreasing function for $t<0$. Assume $k=0$. Since for every $B>0$ $p(B t)=B^{0} p(t)=p(t)$, it follows that $p(t)=$ Const $>0$.

\section{Oscillatory Properties of Solutions}

Corollary 2. Assume that condition (H1) is satisfied. If $x(t)$ is a nontrivial solution of (1.1), $x(a)=x(b)=0$ and $a<b$, then $x^{\prime}(b) \neq 0$ (a simple zero cannot exist on the left of a double zero).

Proof. Let $x^{\prime}(b)=0$, and, without loss of generality, let $x^{\prime \prime}(b)>0$. Then, by Proposition $1 x(t)>0$ for $t<b$. But $x(a)=0, a<b$. The contradiction proves the corollary.

Proposition 4. Let $x(t)$ be a solution of equation (1.1) such that $x(a)=$ $x^{\prime}(b)=0(a<b), x(t) \neq 0$ for $t \in(a, b)$. If condition (H1) is fulfilled, then $x(t)$ vanishes in $(b,+\infty)$.

Proof. Assume that $x(t)$ does not change sign for $t>b$. Without loss of generality, let $x(t)>0, t \geq b$. Multiplying the equation (1.1) by $x(t)$ and integrating from $a$ to $t$, we obtain

$$
\int_{a}^{t} x(s) x^{\prime \prime \prime}(s) d s=-\lambda \int_{a}^{t} x(s) p(s) f(x(s)) d s .
$$

Integrating the first term by parts, we get

$$
x(t) x^{\prime \prime}(t)-x(a) x^{\prime \prime}(a)-\int_{a}^{t} x^{\prime \prime}(s) x^{\prime}(s) d s=-\lambda \int_{a}^{t} x(s) p(s) f(x(s)) d s
$$


or

$$
x(t) x^{\prime \prime}(t)=\frac{1}{2} x^{\prime 2}(t)-\frac{1}{2} x^{\prime 2}(a)-\lambda \int_{a}^{t} x(s) p(s) f(x(s)) d s .
$$

If $t=b$, we obtain

$$
x(b) x^{\prime \prime}(b)=\frac{1}{2} x^{\prime 2}(b)-\frac{1}{2} x^{\prime 2}(a)-\lambda \int_{a}^{b} x(s) p(s) f(x(s)) d s<0 .
$$

Since $x(b)>0$, then $x^{\prime \prime}(b)<0$. Since $x(t)>0$, then (in view of (H1) and (1.1)) $x^{\prime \prime \prime}(t)<0$ and $x^{\prime \prime}(t)$ is strictly decreasing. Thus, $x^{\prime \prime}(t)<0$ for $t>b$ and $x^{\prime}(t)$ is strictly decreasing for $t>b$. Since $x^{\prime}(b)=0$ and $x^{\prime}(t)$ is strictly decreasing for $t>b$, then $x^{\prime}(t)<0$ for $t>b$. Thus, $x(t)$ is strictly decreasing for $t>b$. If two consecutive derivatives of $x(t)$ are negative then $x(t)$ must ultimately be negative. This completes the proof of the proposition.

Proposition 5. Let $x(t)$ be a solution of equation (1.1) such that $x(a)=0$. If conditions $(\mathrm{H} 1),(\mathrm{H} 2)$ and $(\mathrm{H} 3)$ hold, then $x(t)$ vanishes in $(a,+\infty)$.

Proof. Suppose that $x(t)$ does not vanish for $t>a$. Without loss of generality, let $x(t)>0$ for $t>a$. If there exists $b>a$ such that $x^{\prime}(b)=0$, then the proof follows from the Proposition 4 above. Therefore, assume that $x^{\prime}(t)$ does not vanish for $t>a$. Since $x^{\prime}(t)>0$ for $t$ immediately to the right of $a$, it follows that $x^{\prime}(t)>0$ for $t>a$. As $x(t)>0$, then (in view of (H1) and (1.1)), $x^{\prime \prime \prime}(t)<0$ and $x^{\prime \prime}(t)$ is strictly decreasing.

First suppose there exists $t_{1} \geq a$ such that $x^{\prime \prime}\left(t_{1}\right)=0$. Then $x^{\prime \prime}(t)<0$ for $t>t_{1}$. If two consecutive derivatives of $x^{\prime}(t)$ are negative then $x^{\prime}(t)$ must ultimately be negative.

Now assume that $x^{\prime \prime}(t)>0$ for $t>a$. So $x^{\prime}(t)$ is strictly increasing for $t>a$. Integrating equation (1.1) between $t_{0}>a$ and $t$ we obtain

$$
\int_{t_{0}}^{t} x^{\prime \prime \prime}(s) d s=-\lambda \int_{t_{0}}^{t} p(s) f(x(s)) d s
$$

or eliminating nonnegative terms we get

$$
x^{\prime \prime}\left(t_{0}\right)=x^{\prime \prime}(t)+\lambda \int_{t_{0}}^{t} p(s) f(x(s)) d s \geq \lambda \int_{t_{0}}^{t} p(s) f(x(s)) d s .
$$

In view of Proposition 2 and Proposition 3 the integral on the right is unbounded as $t \rightarrow+\infty$, but the left hand side is independent of $t$. This contradiction proves the proposition.

Remark 2. The condition $x(a)=0$ in Proposition 5 is essential, because there exist solutions of equation (1.1) which do not vanish. For example, it is easy to verify, that the function

$$
x(t)=(105 / 8)^{\frac{1}{2}}\left(t-t_{0}\right)^{-\frac{3}{2}}
$$

is a solution of the equation $x^{\prime \prime \prime}=-x^{3}$ which do not vanish. 
Corollary 3. Assume that conditions (H1), (H2) and (H3) are fulfilled. If $x(t)$ is a nontrivial solution of (1.1) and $t=a$ is a zero of $x(t)$, then $x(t)$ has an infinity of simple zeros in $(a,+\infty)$. If $t=a$ is a double zero of $x(t)$, then $x(t)$ does not vanish in $(-\infty, a)$.

\section{Dependence of Zeros on Initial Data}

Proposition 6. Suppose that conditions (H2) and (H3) are fulfilled. If $x(t)$ is a solution of equation (1.1) with $\lambda \equiv 1$, such that $x(0)=x^{\prime}(0)=0, x^{\prime \prime}(0)=$ $\beta_{0} \neq 0$, then every solution of equation (1.1) with arbitrary $\lambda$ which has a double zero at $t=0$ and the second derivative at $t=0$ of the same sign as $\beta_{0}$ can be expressed via solution $x(t)$ as

$$
y(t)=\lambda^{\frac{1}{1-q}} A x\left(A^{(q-1) /(3+k)} t\right)
$$

with initial data $y(0)=y^{\prime}(0)=0, y^{\prime \prime}(0)=\lambda^{\frac{1}{1-q}} A^{(2 q+k+1) /(3+k)} \beta_{0}$, where $A>0$ is a parameter.

Remark 3. We distinguish the cases of $x^{\prime \prime}$ having opposite signs at $t=0$ in Proposition 6 , because if $x(t)$ is a solution of $(1.1)$, then $(-x(t))$ need not to be a solution of (1.1). As an example we present the equation

$$
x^{\prime \prime \prime}=- \begin{cases}3 x^{5}, & x \geq 0, \\ 2 x^{5}, & x<0,\end{cases}
$$

where $f(x)$ satisfies condition (H3) with $q=5$.

Remark 4. A similar statement for higher order Emden-Fowler type autonomous equation can be found in [1].

Proof. The proposition can be proved by direct substitution. So

$$
y^{\prime \prime \prime}(t)=\lambda^{\frac{1}{1-q}} A^{\frac{3 q+k}{3+k}} x^{\prime \prime \prime}\left(A^{\frac{q-1}{3+k}} t\right), \quad f(y(t))=\lambda^{\frac{q}{1-q}} A^{q} f\left(x\left(A^{\frac{q-1}{3+k}} t\right)\right) .
$$

Then

$$
\begin{aligned}
\lambda^{\frac{1}{1-q}} A^{\frac{3 q+k}{3+k}} x^{\prime \prime \prime}\left(A^{\frac{q-1}{3+k}} t\right) & =-\lambda p(t) \lambda^{\frac{q}{1-q}} A^{q} f\left(x\left(A^{\frac{q-1}{3+k}} t\right)\right) \\
& =-\lambda p\left(A^{\frac{q-1}{3+k}} t\right) A^{-k \frac{q-1}{3+k}} \lambda^{\frac{q}{1-q}} A^{q} f\left(x\left(A^{\frac{q-1}{3+k}} t\right)\right) \\
& =\lambda A^{-k \frac{q-1}{3+k}} \lambda^{\frac{q}{1-q}} A^{q} x^{\prime \prime \prime}\left(A^{\frac{q-1}{3+k}} t\right) .
\end{aligned}
$$

Therefore

$$
\lambda^{\frac{1}{1-q}} A^{\frac{3 q+k}{3+k}}=\lambda A^{-k \frac{q-1}{3+k}} \lambda^{\frac{q}{1-q}} A^{q}, \quad \text { or } \quad \lambda^{\frac{1}{1-q}} A^{\frac{3 q+k}{3+k}}=\lambda^{\frac{1}{1-q}} A^{\frac{3 q+k}{3+k}} .
$$

Hence the proof.

Remark 5. Suppose that $f(x)=\kappa x(q=1)$ is a linear function and consider the solution $x(t)$ of equation (1.1) with $\lambda \equiv 1$ which satisfies initial data (1.2). 
Let us denote simple zeros of $x(t)$ to the right from $t_{0}=0$ by $t_{1}, t_{2}, \ldots$ If we change $\beta$, then the points $t_{1}, t_{2}, \ldots$ do not change the location (because of linear dependence of solutions). These points are called conjugate points [3]. If $f(x)$ is not a linear function $(q \neq 1)$, the points $t_{1}, t_{2}, \ldots$ can change their location.

Let $y(t)$ be a nontrivial solution of equation (1.1) with the initial conditions (1.2). Let us denote simple zeros of $y(t)$ to the right from $t_{0}=0$ by $t_{1}(\lambda, \beta)$, $t_{2}(\lambda, \beta), \ldots, t_{i}(\lambda, \beta), \ldots$ and let $t_{i}(1,1)=\sigma_{i}^{+}, t_{i}(1,-1)=\sigma_{i}^{-}$.

Theorem 1. If conditions (H1), (H2) and (H3) hold, then zero functions for $y(t)$ are defined by

$$
\begin{aligned}
& t_{i}(\lambda, \beta)=\sigma_{i}^{+} \lambda^{\frac{-1}{2 q+1+k}} \beta^{\frac{1-q}{2 q+1+k}} \quad \text { for } \beta>0, \\
& t_{i}(\lambda, \beta)=\sigma_{i}^{-} \lambda^{\frac{-1}{2 q+1+k}}(-\beta)^{\frac{1-q}{2 q+1+k}} \quad \text { for } \beta<0 .
\end{aligned}
$$

Proof. First, suppose that $\beta>0$. It follows from Proposition 6, that

$$
t_{i}(\lambda, \beta)=t_{i}(1) / A^{\frac{q-1}{3+k}}=\sigma_{i}^{+} / A^{\frac{q-1}{3+k}} .
$$

Since $\beta=\lambda^{\frac{1}{1-q}} A^{\frac{2 q+1+k}{3+k}}$, then $A=\lambda^{\frac{3+k}{(2 q+1+k)(q-1)}} \beta^{\frac{3+k}{2 q+1+k}}$. Thus, we obtain

$$
t_{i}(\lambda, \beta)=\sigma_{i}^{+} \lambda^{\frac{-1}{2 q+1+k}} \beta^{\frac{1-q}{2 q+1+k}} \quad \text { for } \beta>0 .
$$

Next, suppose that $\beta<0$. It follows from Proposition 6 , that

$$
t_{i}(\lambda, \beta)=t_{i}(-1) / A^{\frac{q-1}{3+k}}=\sigma_{i}^{-} / A^{\frac{q-1}{3+k}} .
$$

Since $\beta=-\lambda^{\frac{1}{1-q}} A^{\frac{2 q+1+k}{3+k}}$, then $A=\lambda^{\frac{3+k}{(2 q+1+k)(q-1)}}(-\beta)^{\frac{3+k}{2 q+1+k}}$. Thus, we obtain

$$
t_{i}(\lambda, \beta)=\sigma_{i}^{-} \lambda^{\frac{-1}{2 q+1+k}}(-\beta)^{\frac{1-q}{2 q+1+k}} \quad \text { for } \beta<0 .
$$

The next two examples illustrate zero functions for certain equations. The first one with super-linear nonlinearity $(q=3>1)$ and the second one with sub-linear nonlinearity $(0<q=3 / 5<1)$.

Example 1. Consider the equation

$$
x^{\prime \prime \prime}=-\lambda t^{2} \begin{cases}100 x^{3}, & x \geq 0, \\ 0.01 x^{3}, & x<0\end{cases}
$$

together with initial data (1.2). Let us obtain $t_{i}(\lambda, \beta)$. By using numerical simulation, we get

$$
\begin{array}{lll}
\sigma_{1}^{+}=1.59729, & \sigma_{2}^{+}=3.44167, & \sigma_{3}^{+}=3.55694, \\
\sigma_{1}^{-}=4.44456, & \sigma_{2}^{-}=4.66656, & \sigma_{3}^{-}=5.19790 .
\end{array}
$$




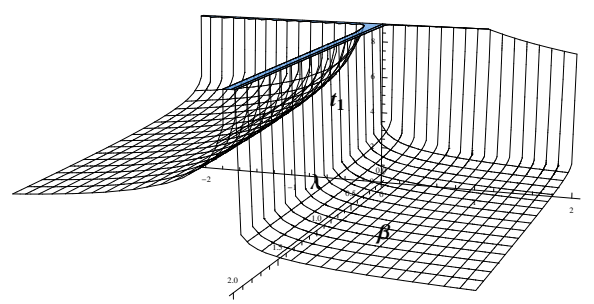

Figure 1. Zero function $t_{1}(\lambda, \beta)$ for equation (4.2).

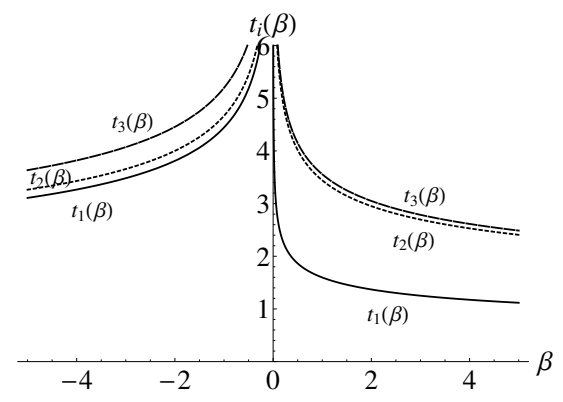

Figure 2. Zero functions for equation (4.2) with $\lambda \equiv 1$. $t_{1}(\beta)-$ solid, $t_{2}(\beta)-$ dotted, $t_{3}(\beta)$ - dashed.

So, by Theorem 1 we get

$$
\begin{aligned}
& t_{1}(\lambda, \beta)=1.59729 \lambda^{-\frac{1}{9}} \beta^{-\frac{2}{9}} \quad \text { for } \beta>0 \text { and } \\
& t_{1}(\lambda, \beta)=4.44456 \lambda^{-\frac{1}{9}}(-\beta)^{-\frac{2}{9}} \quad \text { for } \beta<0, \\
& t_{2}(\lambda, \beta)=3.44167 \lambda^{-\frac{1}{9}} \beta^{-\frac{2}{9}} \quad \text { for } \beta>0 \text { and } \\
& t_{2}(\lambda, \beta)=4.66656 \lambda^{-\frac{1}{9}}(-\beta)^{-\frac{2}{9}} \quad \text { for } \beta<0, \\
& t_{3}(\lambda, \beta)=3.55694 \lambda^{-\frac{1}{9}} \beta^{-\frac{2}{9}} \text { for } \beta>0 \text { and } \\
& t_{3}(\lambda, \beta)=5.19790 \lambda^{-\frac{1}{9}}(-\beta)^{-\frac{2}{9}} \quad \text { for } \beta<0 .
\end{aligned}
$$

See Figure 1, where graph of $t_{1}(\lambda, \beta)$ is presented. Graphs of some zero functions for equation (4.2) with $\lambda \equiv 1$ are presented in Figure 2.

Example 2. Consider the equation

$$
x^{\prime \prime \prime}=-\lambda t^{4} \begin{cases}x^{\frac{3}{5}}, & x \geq 0 \\ 7 x^{\frac{3}{5}}, & x<0\end{cases}
$$

together with initial data (1.2). Let us obtain $t_{i}(\lambda, \beta)$. By using numerical simulation, we get

$$
\begin{array}{lll}
\sigma_{1}^{+}=2.52405, & \sigma_{2}^{+}=2.99564, & \sigma_{3}^{+}=4.12060 \\
\sigma_{1}^{-}=1.84413, & \sigma_{2}^{-}=3.23205, & \sigma_{3}^{-}=3.68724 .
\end{array}
$$




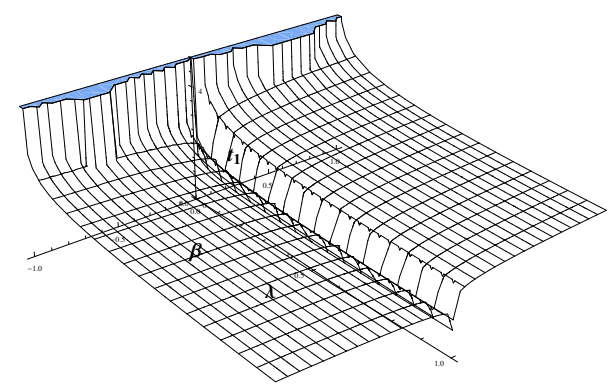

Figure 3. Zero function $t_{1}(\lambda, \beta)$ for equation (4.3).

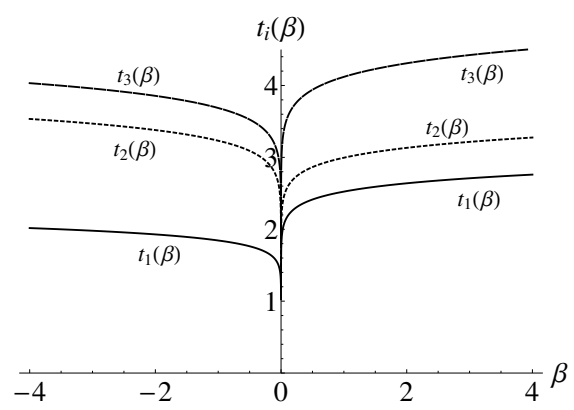

Figure 4. Zero functions for equation (4.3) with $\lambda \equiv 1$. $t_{1}(\beta)-$ solid, $t_{2}(\beta)-$ dotted, $t_{3}(\beta)$ - dashed.

So, by Theorem 1 we get

$$
\begin{aligned}
& t_{1}(\lambda, \beta)=2.52405 \lambda^{-\frac{5}{31}} \beta^{\frac{2}{31}} \quad \text { for } \beta>0 \quad \text { and } \\
& t_{1}(\lambda, \beta)=1.84413 \lambda^{-\frac{5}{31}}(-\beta)^{\frac{2}{31}} \quad \text { for } \beta<0, \\
& t_{2}(\lambda, \beta)=2.99564 \lambda^{-\frac{5}{31}} \beta^{\frac{2}{31}} \quad \text { for } \beta>0 \quad \text { and } \\
& t_{2}(\lambda, \beta)=3.23205 \lambda^{-\frac{5}{31}}(-\beta)^{\frac{2}{31}} \quad \text { for } \beta<0, \\
& t_{3}(\lambda, \beta)=4.12060 \lambda^{-\frac{5}{31}} \beta^{\frac{2}{31}} \quad \text { for } \beta>0 \quad \text { and } \\
& t_{3}(\lambda, \beta)=3.68724 \lambda^{-\frac{5}{31}}(-\beta)^{\frac{2}{31}} \quad \text { for } \beta<0 .
\end{aligned}
$$

See Figure 3, where graph of $t_{1}(\lambda, \beta)$ is presented. Graphs of some zero functions for equation (4.3) with $\lambda \equiv 1$ are presented in Figure 4.

\section{Conclusions}

As we can see from the previous examples, the behavior (monotony and concavity) of the zero functions for the super-linear case $(q>1)$ substantially differs from the sub-linear case $(0<q<1)$. In the case when $\lambda \equiv 1$ zero functions are concave upwards for the super-linear case and concave downwards for the 
sub-linear case; for super-linear case zero functions are increasing for $\beta<0$ and decreasing for $\beta>0$; for sub-linear case zero functions are decreasing for $\beta<0$ and increasing for $\beta>0$.

\section{References}

[1] I.V. Astashova. Application of dynamical systems to the study of asymptotic properties of solutions to nonlinear higher-order differential equations. J. Math. Sci., 126:1361-1391, 2005.

[2] L. Erbe. Existence of oscillatory solutions and asymptotic behavior for a class of third order linear differential equations. Pacific J. Math., 64(2):369-385, 1976. http://dx.doi.org/10.2140/pjm.1976.64.369.

[3] M. Hanan. Oscillation criteria for third order linear differential equation. Pacific J. Math., 11:919-944, 1961. http://dx.doi.org/10.2140/pjm.1961.11.919.

[4] J. W. Heidel. Qualitative behavior of solutions of a third order nonlinear differential equation. Pacific J. Math., 27:507-526, 1968. http://dx.doi.org/10.2140/pjm.1968.27.507.

[5] F. Sadyrbaev and A. Gritsans. Nonlinear spectra for parameter dependent ordinary differential equations. Nonlinear Anal. Model. Control, 12(2):253-267, 2007.

[6] S. Smirnov. On some spectral properties of third order nonlinear boundary value problems. Math. Model. Anal., 17(1):78-89, 2012. http://dx.doi.org/10.3846/13926292.2012.645076.

[7] N.I. Vasilyev and Yu.A. Klokov. Foundations of the Theory of Boundary Value Problems for Ordinary Differential Equations. Zinatne, Riga, 1978.

[8] P. Waltman. Oscillation criteria for third order nonlinear differential equations. Pacific J. Math., 18:385-389, 1966. http://dx.doi.org/10.2140/pjm.1966.18.385. 